\title{
ON THE BOUNDEDNESS OF SUBSEQUENCES OF VILENKIN-FEJÉR MEANS ON THE MARTINGALE HARDY SPACES
}

\author{
LARs-Erik Persson, George Tephnadze and Georgi Tutberidze
}

Abstract. In this paper we characterize subsequences of Fejér means with respect to Vilenkin systems, which are bounded from the Hardy space $H_{p}$ to the Lebesgue space $L_{p}$, for all $0<$ $p<1 / 2$. The result is in a sense sharp.

Mathematics subject classification (2010): 42C10, 42B25.

Keywords and phrases: Vilenkin system, Vilenkin group, Vilenkin-Fejér means, martingale Hardy space, maximal operator, Vilenkin-Fourier series.

\section{REFERENCES}

[1] G. N. Agaev, N. Ya. Vilenkin, G. M. Dzafarly and A. I. Rubinshtein, Multiplicative systems of functions and harmonic analysis on zero-dimensional groups, Baku, Ehim, 1981 (in Russian).

[2] I. Blahota, G. GÁt And U. Goginava, Maximal operators of Fejér means of double VilenkinFourier series, Colloq. Math. J., 107 (2007), no. 2, 287-296.

[3] I. Blahota, G. GÁt And U. Goginava, Maximal operators of Fejér means of Vilenkin-Fourier series, JIPAM. J. Inequal. Pure Appl. Math. 7 (2006), 1-7.

[4] I. Blahota And G. TePhnadZE, Strong convergence theorem for Vilenkin-Fejér means, Publ. Math. Debrecen, 85, 1-2 (2014), 181-196.

[5] I. Blahota, K. Nagy, L.-E. Persson, G. Tephnadze, A sharp boundedness result concerning some maximal operators of partial sums with respect to Vilenkin systems, Georgian Math., J., DOI: https://doi.org/10.1515/gmj-2018-0045.

[6] N. J. FUJII, A maximal inequality for $H_{1}$ functions on the generalized Walsh-Paley group, Proc. Amer. Math. Soc. 77 (1979), 111-116.

[7] G. GÁt, Cesáro means of integrable functions with respect to unbounded Vilenkin systems, J. Approx. Theory 124 (2003), no. 1, 25-43.

[8] U. GoginaVA, Maximal operators of Fejér means of double Walsh-Fourier series, Acta Math. Hungar. 115 (2007), no. 4, 333-340.

[9] U. Goginava And K. NAGY, On the maximal operator of Walsh-Kaczmarz-Fejér means, Czechoslovak Math. J., 61 (2011), no. 3, 673-686.

[10] J. PÁL And P. Simon, On a generalization of the concept of derivative, Acta Math. Acad. Sci. Hungar. 29 (1977), no. 1-2, 155-164.

[11] L.-E. PERSSON AND G. TEPHNADZE, A sharp boundedness result concerning some maximal operators of Vilenkin-Fejér means, Mediterr. J. Math., 13, 4 (2016) 1841-1853.

[12] F. SCHIPP, Certain rearrangements of series in the Walsh series, Mat. Zametki, 18 (1975), $193-201$.

[13] P. Simon, Cesáro summability with respect to two-parameter Walsh systems, Monatsh. Math., 131, 4 (2000), 321-334.

[14] P. Simon, Investigations with respect to the Vilenkin system, Ann. Univ. Sci. Budapest. Eötvös Sect. Math., 28 (1985), 87-101.

[15] B. SMITH, A strong convergence theorem for $H_{1}(T)$, Lecture Notes in Math., 995, Springer, Berlin, 1994, 169-173.

[16] G. TePhnAdzE, On the maximal operator of Vilenkin-Fejér means, Turk. J. Math, 37, (2013), 308318. 
[17] G. TephnAdZE, On the maximal operators of Vilenkin-Fejér means on Hardy spaces, Math. Inequal. Appl., 16, (2013), no. 2, 301-312.

[18] G. TephnAdZE, On the Vilenkin-Fourier coefficients, Georgian Math. J., 20, 1 (2013), 169-177.

[19] G. TEPHNADZE, On the convergence of partial sums with respect to Vilenkin system on the martingale Hardy spaces, J. Contemp. Math. Anal., 53, 5, (2018) 294-306.

[20] N. YA. ViLENKIn, On a class of complete orthonormal systems, Izv. Akad. Nauk. U.S.S.R., Ser. Mat., 11 (1947), 363-400.

[21] F. WeISZ, Martingale Hardy spaces and their applications in Fourier Analysis, Springer, BerlinHeideiberg-New York, 1994.

[22] F. WeIsz, Hardy spaces and Cesáro means of two-dimensional Fourier series, Bolyai Soc. Math. Studies, (1996), 353-367.

[23] F. Weisz, Cesáro summability of one- and two-dimensional Walsh-Fourier series, Anal. Math. 22 (1996), no. 3, 229-242.

[24] F. WeIsz, Weak type inequalities for the Walsh and bounded Ciesielski systems, Anal. Math. 30 (2004), no. 2, 147-160. 